\title{
BMJ Open Assessing the transparency of informed consent in feasibility and pilot studies: a single-centre quality assurance study protocol
}

Mohammed I Khan (D) , ${ }^{1}$ Matthew Holek, ${ }^{1}$ Faris Bdair, ${ }^{1}$ Lawrence Mbuagbaw (D) , ${ }^{1,2}$ Sandra M Eldridge ${ }^{3}$ Claire L Chan, ${ }^{4}$ Michael J Campbell (1D , ${ }^{5}$ Christine M Bond, ${ }^{6}$ Sally Hopewell, ${ }^{7}$ Gillian A Lancaster, ${ }^{8}$ Lehana Thabane ${ }^{1,2}$

To cite: Khan Ml, Holek M, Bdair F, et al. Assessing the transparency of informed consent in feasibility and pilot studies: a singlecentre quality assurance study protocol. BMJ Open 2020;10:e036226. doi:10.1136/ bmjopen-2019-036226

- Prepublication history and additional material for this paper are available online. To view these files, please visit the journal online (http://dx.doi. org/10.1136/bmjopen-2019036226).

Received 06 December 2019 Revised 20 March 2020 Accepted 07 May 2020

Check for updates

(C) Author(s) (or their employer(s)) 2020. Re-use permitted under CC BY-NC. No commercial re-use. See rights and permissions. Published by BMJ.

For numbered affiliations see end of article.

Correspondence to

Dr Lehana Thabane;

thaban!@mcmaster.ca

\section{ABSTRACT}

Introduction Pilot/feasibility studies assess the feasibility of conducting a larger study. Although researchers ought to communicate the feasibility objectives to their participants, many research ethics guidelines do not comment on how informed consent applies to pilot studies. It is unclear whether researchers and research ethics boards clearly communicate the purpose of pilot studies to participants consenting.

The primary objective of this study is to assess whether pilot/feasibility studies submitted for ethics approval to a research ethics board transparently communicate the purpose of the study to participants through their informed consent practice. A highly transparent consent practice entails the consent documents communicate: (1) the term 'pilot' or 'feasibility' in the title; (2) the definition of a pilot/ feasibility study; (3) the primary objectives of the study are to assess feasibility; (4) the specific feasibility objectives; and (5) the criteria for the study to successfully lead to the main study. The secondary objectives are to assess whether there is a difference between submitted and revised versions of the consent documents (revisions are made to obtain research ethics approval), to determine factors associated with transparent consent practices and to assess the consistency with which pilot and feasibility studies assess feasibility outcomes as their primary objectives. Methods and analysis This is a retrospective review of informed consent information for pilot/feasibility studies submitted to the Hamilton integrated Research Ethics Board, Canada. We will look at submitted and revised consent documents for pilot/feasibility studies submitted over a 14-year period. We will use descriptive statistics to summarise data, reporting results as percentages with $95 \% \mathrm{Cls}$, and conduct logistic regression to determine characteristics associated with transparent consent practices.

Ethics and dissemination The study protocol was approved by the Hamilton integrated Research Ethics Board, and the results of this study will be submitted for publication in a peer-reviewed journal.

\section{INTRODUCTION}

A feasibility study is a preliminary investigation done to assess the feasibility of a

\section{Strengths and limitation of this study}

- It is the first known study to assess whether researchers are clearly communicating the purposes of their pilot/feasibility studies to participants.

- It raises issues that remain unaddressed in many research ethics guidelines.

- It will be used to develop guidelines on how future pilot/feasibility studies can best maintain high standards of informed consent.

- Conducted at one centre, in Canada, with a limited sample size, looking at studies submitted in English.

subsequent larger study. ${ }^{1}$ A pilot study is a type of feasibility study in which the future study is implemented, or partially implemented, on a small scale to assess feasibility. ${ }^{2}$ Evidence suggests that researchers use the terms 'pilot' and 'feasibility' without distinction, ${ }^{3}$ and because we are interested in all studies assessing feasibility, we will make no distinction between pilot and feasibility studies in our investigation.

The three main reasons for conducting a pilot study are to assess feasibility questionsrelated to process, resources and management. ${ }^{45}$ Process-related objectives assess the feasibility of steps that need to be taken to ensure the success of the main study, like recruitment, retention and understanding of data collection tools. ${ }^{4-6}$ Resource-related objectives assess issues of time, capacity and other resources that could occur in the main study. $^{4-6} \quad$ Management-related objectives assess human and data management problems that can arise in the main study, like identifying challenges that study personnel would face. $^{4-6}$

However, an ethical issue remains largely unaddressed: the obligation of researchers to communicate the nature and objectives 
of pilot studies to participants of these studies, when obtaining consent. ${ }^{6}$ This communication is essential in maintaining the rights of research participants, credibility of researchers and trust between researchers and participants, as some pilot studies may not proceed to future studies. For instance, Lancaster $e t \mathrm{al}^{7}$ found that about half of pilot studies published indicated that further study was needed, and other studies have found that the proportion of pilot studies that lead to larger studies is between $5 \%$ and 24\%.$^{38}$ Although in 2010, Thabane et at developed recommendations for communicating the objectives and necessary information about pilot studies to participants, there is a paucity of data and conversation on this issue in the literature. Research ethics guidelines including the Nuremberg Code, ${ }^{9}$ Declaration of Helsinki, ${ }^{10}$ the Belmont Report, ${ }^{11}$ International Conference on Harmonization Good Clinical Practice ${ }^{12}$ and the International Ethical Guidelines for Health-related Research Involving Humans ${ }^{13}$ do not comment on pilot studies. ${ }^{6}$ In 2018, the Tri-Council Policy Statement $2^{14}$ (TCPS2) was revised to include informed consent practices in pilot studies. However, other research ethics guidelines need to be updated to address pilot studies, because they are a special case in which evidence suggests that the reporting and methods are inadequately approached. ${ }^{3}$ 6-8 With limited guidelines on informed consent in pilot studies, can we expect researchers and research ethics boards to adequately address this issue?

The revised TCPS2 guidelines for informed consent in pilot studies state that researchers have an ethical responsibility to communicate the purpose and nature of pilot studies to participants when seeking consent. ${ }^{14}$ Currently, we do not know if researchers are effectively communicating the feasibility nature of pilot studies to their participants and obtaining an acceptable level of informed consent. After conducting a comprehensive literature search of studies assessing practices of informed consent in pilot studies, we concluded that this issue has not been empirically investigated in the literature.

Participants are entitled to have honest and complete information about the studies they are invited to participate in, with some exceptions, like when deception or waiver of consent is necessary to conduct a study, and when such modifications of informed consent are approved by an ethics committee. However, depriving participants of a complete understanding of the pilot studies they are invited to participate in has ethical implications. Thus, it is imperative that the issue of informed consent in pilot studies is investigated. The results of this study will help to quantify the degree of this issue at one centre and could lead to recommendations for all research ethics boards to ensure high standards of informed consent are applied to future pilot studies.

We hypothesise that a significant number of approved pilot studies across various research ethics boards do not communicate clearly the purpose of the study to their participants. This study will be the first attempt to obtain empirical evidence on current practice regarding informed consent processes in pilot and feasibility studies.

\section{Study objectives}

Our primary objective is to assess whether pilot studies submitted to the Hamilton integrated Research Ethics Board (HiREB), Canada, transparently communicate the purpose of the study to participants through their informed consent practice. A highly transparent informed consent practice entails the patient information documents and/or consent forms effectively communicate: (1) the term 'pilot' or 'feasibility' in the title of the study; (2) the definition of a pilot or feasibility study; (3) the objectives or purpose of the study are stated clearly as assessing feasibility; (4) the specific feasibility objectives of the study and (5) the criteria for the feasibility study to successfully lead to the main study. Thus, the primary objective is to assess whether pilot studies submitted to the HiREB for research ethics approval communicate the preceding characteristics within their informed consent forms or patient information documents. The HiREB was chosen as the site for this study, as it is McMaster University's primary research ethics board. McMaster University is Canada's most research-intensive, medical doctoral university, ${ }^{15}$ so the HiREB is likely to see a wide variety of pilot studies.

Our secondary objectives are: (1) to assess whether there is a difference between the submitted and revised versions of the informed consent forms and patient information documents (revisions are made in order to obtain research ethics approval), specifically in addressing the issues and criterion discussed in the primary objective; (2) to determine methodological characteristics associated with increased reporting or inclusion of the criterion discussed in the primary objectives within the original informed consent forms and patient information documents; and (3) to assess the consistency with which feasibility and pilot studies assess feasibility outcomes as their primary objectives.

\section{METHODS AND ANALYSIS}

\section{Sample selection}

Inclusion criteria

All pilot or feasibility studies submitted to the HiREB that use the term 'pilot' or 'feasibility' in their title, between January 2006 and December 2019 inclusive, which require participant consent, including both randomised and nonrandomised pilot or feasibility studies.

\section{Exclusion criteria}

All pilot or feasibility studies that have a waiver of consent.

\section{Sample size}

We used the estimation method for a single proportion, a statistical approach used to determine appropriate sample size based on inputted values for a proportion, confidence interval (CI), and margin of error to determine our 
sample size. ${ }^{16}$ We require 196 subjects if the true proportion of pilot studies that use the term 'pilot' or 'feasibility' in the title of the study, as stated on the consent form, is 0.5 , which will be estimated with a $95 \% \mathrm{CI}$ of about 0.43 to 0.57 (see the online supplementary appendix 1 ). If more than 196 studies meet the inclusion criteria, then we will include all of them in the analysis to decrease the width of the CI. If fewer than 196 studies meet the inclusion criteria, then based on the same proportion and a $95 \%$ CI of 0.40 to 0.60 , a sample of 96 will be sufficient to address the objectives of the study (see the online supplementary appendix 1).

\section{Data collection}

Anonymised data will be extracted from the online HiREB database of studies using the data abstraction form (see the online supplementary appendix 2) and entered into a password-protected Microsoft Office Excel file, on a password-protected computer, within a locked office at the study site. Data will be extracted by two independent reviewers for a subset of the studies and a $\kappa$ value with a 95\% CI will be calculated to assess agreement. Reviewers will receive the same training to ensure that the data extraction tool is used consistently. All disagreements will be resolved by a third party and reasons for disagreements will be identified and used to update standard operating procedures to support consistency in future coding.

To address the primary objective of the study, we will collect information on both the initially submitted and revised participant consent forms and patient information documents specifically: (1) whether the term 'pilot' or 'feasibility' is in the title of the study; (2) whether the definition of a pilot or feasibility study is stated; (3) whether the objectives or purpose of the study are stated clearly as to assess feasibility; (4) whether the specific feasibility objectives of the study are stated and (5) the criteria for the feasibility study to successfully lead to the main study are stated.

To address the secondary objectives, we will also collect information on the methodology, design and protocol of the pilot studies specifically: (1) whether the studies were randomised or not; (2) whether the data collected were qualitative or quantitative (or both); (3) the desired sample size; (4) the year of original submission to the HiREB; (5) whether the study was reviewed by HiREB panel A, B or unidentified; (6) whether the study was observational or interventional; (7) the study's source of funding; (8) whether the study was approved or not; (9) whether the study was labelled a 'pilot' or 'feasibility' study (or both); and (10) the specific feasibility objectives of the studies.

Please see the online supplementary appendix 2 for the full data abstraction form.

\section{Data analysis}

Descriptive statistics will be used to summarise the characteristics of the studies, including information on the methodology, design and protocol of the studies. These statistics will be reported as counts (percent) or mean (SD) and presented in tables. Results from the primary analysis will be used to determine the percentages of studies in the sample that reported each item (from the primary objective) and the average number of items reported, with the corresponding $95 \%$ CI. For the secondary objectives, we will use logistic regression to analyse the data and assess the proportion of studies labelled 'pilot' or 'feasibility' primarily assessing feasibility, with a 95\% CI. Please see the table in the online supplementary appendix 3 for details and a full summary of the analysis plan. All analyses will be performed using SAS V.9.2.

Please see the online supplementary appendix 3 for a summary of the study objectives and data analysis.

\section{Patient and public involvement}

No patients were involved in the development of this study.

\section{ETHICS AND DISSEMINATION}

\section{Ethics approval and ethical concerns}

The study protocol was approved by the HiREB (project no.: $7071 \mathrm{C}$ ), which granted a waiver of consent for the research team to access the files and consent forms from pilot and feasibility studies submitted to the HiREB.

\section{Dissemination}

The results of this study and any ancillary studies will be submitted for publication in peer-reviewed journals, independent of their statistical significance and in a timely manner. Furthermore, the results of this study will be used to develop guidelines on the best practices for the development of consent forms in pilot studies. Although this is a single-centre study, if issues of transparency exist, then they likely extend to other research ethics boards and by establishing some evidence-based guideline, we may be able to help researchers and research ethics boards maintain higher standards of informed consent in pilot studies. Our findings will also be made available through conference abstracts, statistical workshops, online sources and to research ethics boards. The complete data set will only be accessible by the research team, but requests for the use of this information in secondary publications and collaborations are encouraged.

\section{DISCUSSION}

Challenges surrounding informed consent have existed for many years. With the advent of new research methods, like pilot, biobank and online studies, new challenges emerge in the consent process. ${ }^{617-19}$ Some of these challenges, like issues related to consent in biobanks, raise concerns that truly informed consent is an ethical aspiration rather than an ethical requirement. ${ }^{17}$

In making decisions on informed consent, it is important to consider its purpose. Dickert identified seven functions of informed consent: (1) providing 
transparency; (2) allowing control and authorisation; (3) promoting concordance with participants' values; (4) protecting and promoting welfare interests; (5) promoting trust; (6) satisfying regulatory requirements and (7) promoting integrity in research. The first four functions apply more to the individual participants, whereas the latter three focus on policy and systemic functions, with each function existing to a degree. ${ }^{20}$ Innovative approaches to informed consent suggest making context-dependent informed consent forms, in which certain information like that which is deliberately communicated outside the consent documents is omitted.$^{20}$ However, regardless of context, consent forms ought to convey the purpose of the research being conducted, as they are essential in fulfilling five of the seven functions of informed consent: providing transparency, promoting concordance with participants' values, promoting trust, satisfying regulatory requirements and promoting integrity in research. Thus, when it comes to pilot studies, their purpose, although complex and nuanced, needs to be communicated to participants in order to maintain the functions of informed consent.

If researchers are not clearly communicating the purposes of their pilot studies to participants, then there is great potential for risk and unquantifiable harm, as study participants' rights can be violated, researchers' credibility damaged and trust between participants and researchers broken. For example, if a participant of a pilot study discovers that they were lied to about the objectives of the research they agreed to participate in, they could feel betrayed; question the integrity of researchers; decide not to participate in future studies; question the integrity of research and evidenced-based medicine; and even take legal action against researchers, research sponsors or research ethics committees. This single-centre study will be the first to empirically measure whether researchers and research ethics boards are applying the general principles of informed consent, as highlighted by various research ethics guidelines, ${ }^{9-14}$ to pilot studies. Regardless of the results of this study, it is important to acknowledge that research ethics guidelines need an update, one that addresses informed consent in pilot studies specifically. We urge other institutions to conduct similar quality assurance projects to better quantify issues surrounding informed consent in pilot studies and encourage a timely change to research ethics guidelines.

\footnotetext{
Author affiliations

${ }^{1}$ Biostatistics Unit, St Joseph's Healthcare Hamilton, Hamilton, Ontario, Canada ${ }^{2}$ Department of Health Research Methods, Evidence and Impact, McMaster University, Hamilton, Ontario, Canada

${ }^{3}$ Barts and The London Pragmatic Clinical Trials Unit, Queen Mary University of London, London, UK

${ }^{4}$ Centre for Primary Care and Public Health, Queen Mary University of London, London, UK

${ }^{5}$ School of Health and Related Research, The University of Sheffield, Sheffield, UK ${ }^{6}$ Institute of Applied Health Sciences, University of Aberdeen, Aberdeen, UK
}

${ }^{7}$ Centre for Statistics in Medicine, Nuffield Department of Orthopaedics, Rheumatology and Musculoskeletal Sciences, University of Oxford, Oxford, UK ${ }^{8}$ School of Primary, Community and Social Care; Keele Clinical Trials Unit, Keele University, Newcastle under Lyme, UK

\section{Twitter Christine M Bond @christinebond20}

Contributors MIK and LT conceived and designed the study; obtained approval to conduct the study and access necessary documents; and designed the data abstraction tool. MH, FB, LM, SE, CLC, MJC, CB, SH, and GL contributed to the development of the data extraction form. LT provided statistical expertise. MIK drafted the manuscript. All authors revised and approved the final manuscript and contributed their scholarly input.

Funding The authors have not declared a specific grant for this research from any funding agency in the public, commercial or not-for-profit sectors.

Competing interests None declared.

Patient and public involvement Patients and/or the public were not involved in the design, or conduct, or reporting, or dissemination plans of this research.

Patient consent for publication Not required.

Provenance and peer review Not commissioned; externally peer reviewed.

Open access This is an open access article distributed in accordance with the Creative Commons Attribution Non Commercial (CC BY-NC 4.0) license, which permits others to distribute, remix, adapt, build upon this work non-commercially, and license their derivative works on different terms, provided the original work is properly cited, appropriate credit is given, any changes made indicated, and the use is non-commercial. See: http://creativecommons.org/licenses/by-nc/4.0/.

\section{ORCID iDs}

Mohammed I Khan http://orcid.org/0000-0003-4116-0097

Lawrence Mbuagbaw http://orcid.org/0000-0001-5855-5461

Michael J Campbell http://orcid.org/0000-0003-3529-2739

\section{REFERENCES}

1 Everitt B. Medical statistics from a to Z: a guide for clinicians and medical students. Cambridge: Cambridge University Press, 2006.

2 Eldridge SM, Lancaster GA, Campbell MJ, et al. Defining feasibility and pilot studies in preparation for randomised controlled trials: development of a conceptual framework. PLoS One 2016;11:e0150205.

3 Arain M, Campbell MJ, Cooper CL, et al. What is a pilot or feasibility study? A review of current practice and editorial policy. BMC Med Res Methodol 2010;10:67.

4 Van Teijlingen ER, Rennie AM, Hundley V, et al. The importance of conducting and reporting pilot studies: the example of the Scottish births survey. J Adv Nurs 2001;34:289-95.

5 Teijlingen Evan, Hundley V. The importance of pilot studies. Nurs Stand 2002;16:33-6.

6 Thabane L, Ma J, Chu R, et al. A tutorial on pilot studies: the what, why and how. BMC Med Res Methodol 2010;10:1.

7 Lancaster GA, Dodd S, Williamson PR. Design and analysis of pilot studies: recommendations for good practice. J Eval Clin Pract 2004;10:307-12.

8 Shanyinde M, Pickering RM, Weatherall M. Questions asked and answered in pilot and feasibility randomized controlled trials. BMC Med Res Methodol 2011;11:117.

$9 \mathrm{NIH}$. The Nuremberg code. Available: https://history.nih.gov/ research/downloads/nuremberg.pdf [Accessed Nov 2019].

10 The World Medical Association. Declaration of Helsinki, 2013. Available: https://www.wma.net/policies-post/wma-declaration-ofhelsinki-ethical-principles-for-medical-research-involving-humansubjects/ [Accessed Nov 2019].

11 HHS.gov. The Belmont report.ethical principles and guidelines for the protection of human subjects of research, 1979. https://www.hhs. gov/ohrp/regulations-and-policy/belmont-report/read-the-belmontreport/index.html

12 WHO. ICH Harmonized Tripartite Guideline - Guideline for Good Clinical Practice E6(R1), 1996. Available: https://apps.who.int/ medicinedocs/documents/s22154en/s22154en.pdf [Accessed Nov 2019].

13 The Council for International Organizations of Medical Sciences (CIOMS), World Health Organization. International ethical guidelines for health-related research involving humans, 2016. Available: 
https://cioms.ch/wp-content/uploads/2017/01/WEB-CIOMSEthicalGuidelines.pdf [Accessed Nov 2019].

14 Canadian Institutes of Health Research, Natural Sciences and Engineering Research Council of Canada, Social Sciences and Humanities Research Council. Tri-Council Policy Statement .Ethical Conduct for Research Involving Humans. Government of Canada, 2018. http://www.pre.ethics.gc.ca/eng/documents/tcps2-2018-eninteractive-final.pdf

15 Research Infosource Inc. Canada's Top 50 Research Universities 2018. Top Research Universities by Tier - Winners Circle., 2018. Available: https://researchinfosource.com/top-50-researchuniversities/2018/top-research-universities-by-tier [Accessed Oct 2019].
16 Machin D, Campbell MJ, Tan SB, et al. Sample sizes for clinical, laboratory and epidemiological studies. 138. Hoboken,NJ: WileyBlackwell, 2018.

17 Beskow LM, Weinfurt KP. Exploring Understanding of "Understanding": The Paradigm Case of Biobank Consent Comprehension. Am J Bioeth 2019;19:6-18.

18 Grady C, Cummings SR, Rowbotham MC, et al. Informed consent. N Engl J Med 2017;376:856-67.

19 Henderson GE. Is informed consent broken? Am J Med Sci 2011;342:267-72.

20 Dickert NW, Eyal N, Goldkind SF, et al. Reframing consent for clinical research: a function-based approach. Am J Bioeth 2017;17:3-11. 